\title{
Threshold Based Adaptive Power-Law Applications in Image Enhancement
}

\author{
T. Romen Singh \\ Department of I.T, School of \\ Technology, Assam University, \\ Silchar - 788011, Assam, India
}

\author{
Sudipta Roy \\ Department of I.T, School of \\ Technology, Assam University, \\ Silchar - 788011, Assam, India
}

\author{
Kh. Manglem Singh \\ Department of Computer Sc. and \\ Engineering, NIT, Manipur \\ Imphal -795001, Manipur, India.
}

\begin{abstract}
This paper presents a spatial domain threshold based adaptive power-law applications (TAPLA) in image enhancement technique in which adaptation is carried out with local thresholds. This is an improved version of Adaptive Power-law Transformations (APLT) [14] in which adaptation is carried out with local means. The computational time of APLT is windowsize dependent to find local mean while the TAPLA is independent of window-size to find local means, which are used to determine the local threshold values. Window-size independent of computational time is due to use of integral average image as prior process to find local mean. Like APLT, TAPLA can control the enhancement factors such as contrast, brightness and sharpness/smoothness with a proper choice of parameters through a single function. This method can be applied on both the grey scale and color images. In the case of color images, each channel is considered separately. TAPLA outperforms better than APLT in image quality as well as in computational time.
\end{abstract}

\section{Keywords}

Adaptive, power-law, Image enhancement, Contrast, Transformations, Image sharpening, Artifact, integral average image.

\section{INTRODUCTION}

Image enhancement is a process of improving the quality of an image for visual perception by human beings and to make images more suitable for analysis. It is an important operation in the general fields of image processing and computer vision. It is also important in low level vision applications. Enhancement operation is a task in which a set of pixel values of one image is transformed to a new set of pixel values of a new image which is visually more pleasing and is also more suitable for analysis. Machine vision has many important applications of digital images that are captured in low contrast conditions. These images often encounter serious problems in recognition systems. Hence contrast and edge enhancement is an important part to solve such problems. How to enhance the contrast is a vital factor in image recognition problem, and many methods for improving the image quality in contrast have been proposed.

Contrast enhancement is important in medical image applications due to the fact that visual examination of medical images is essential in the diagnosis of many diseases such as chest radiography and mammography [3], [6]. The image contrast is inherently low due to the small differences in the X-ray attenuation coefficients

Histogram equalization [1] and Adaptive Contrast Enhancement ACE [12][13][30] are the well known contrast enhancement methods for poor intensity distribution images. In addition to this, edge enhancement is also important in image recognition system. Multi scale edge enhancement using the wavelet transform [2] is a way to enhance the contrast by enhancing the edges in scale space since edges play a fundamental role in image understanding. Curvelet transformation is well-adapted to represent images containing edges, and it works well for edge enhancement [5]. Curvelet coefficients can be modified in order to enhance edges in an image. Most of the methods are dedicated to a single purpose. For example, contrast enhancement technique fails in edge enhancement cases while edge enhancement technique fails in contrast enhancement case. Power-Law Transformations [1] is also a powerful contrast stretching function. T.R Singh et al [14] propose Adaptive Power-Law Transformations for image enhancement through contrast stretching as well as edges sharpening. It may cause over enhancement in the non-edges region sometimes. So as to solve this problem, we propose Threshold Based Adaptive Powe-Law Applications in image enhancement which is an advanced version of ALPT using a threshold value[15] to control the edge region.

Most of the current techniques are developed for grey scale images. The generalization of these techniques to color images is not straight forward. Unlike grey scale images, there are some factors in color images like hue, which needs to be properly taken care of for enhancement. Hue, saturation and intensity are the attributes of color [1]. Hue is that attribute of color which decides what kind of color it is, i.e., a red or an orange. One needs to improve the visual quality of an image without distorting attributes of color of the image. Several algorithms are available for contrast enhancement for grey scale images, which change the grey values of pixels depending on the criteria for enhancement without taking care about the color attributes like hue. In color image enhancement, it must preserve the three attributes of color images. Sarif Kumar Naik and C. A. Murthy [9] proposed a color image hue preserving enhancement technique where hue unaltered transformation of the image data from RGB space to other color spaces such as LHS, HSI, YIQ, HSV, etc. is done. The proposed technique TAPLA is also applicable in color images. For color images, TAPLA is applied channel wise.

This paper is organized as follows. Section 2 describes integral average image as a prior process to find local mean, which does not depend on local window size. Section 3 describes the technique of finding threshold value by T.R Singh et al [15 ], Section 4 describes image transformations and adaptive transformation like APLT and ACE, Session 5 describes the proposed Technique TAPLA and Session 6 gives the experimental results followed by conclusions in Section 7.

\section{INTEGRAL IMAGES}

An integral image $S$ of an input image I is defined as the image in which the intensity at a pixel position is equal to the sum of the intensities of all the pixels above and to the left of that position in the original image and it can be defined as 
$\mathrm{S}(\mathrm{x}, \mathrm{y})=\sum_{\mathrm{i}=1}^{\mathrm{x}} \sum_{\mathrm{j}=1}^{\mathrm{y}} \mathrm{I}(\mathrm{x}, \mathrm{y}) \mathrm{s} \equiv$

Integral sum image representation has a problem of large value of sum. If the image dimension is very large the sum value at locations farther from origin will be very large and may not have enough memory space to store. In order to solve this problem, the sum value is represented as average of the intensities within that rectangular area up to the pixel position which are under consideration.

\subsection{Integral Average Image}

The integral average image $g$ of an input image I is defined as the image in which the intensity at a pixel position is equal to the average of the intensities of all the pixels above and to the left of that position in the original image and it can be defined as

$\mathrm{g}(\mathrm{x}, \mathrm{y})=\frac{1}{\mathrm{x} \times \mathrm{y}} \sum_{\mathrm{i}=1}^{\mathrm{x}} \sum_{\mathrm{j}=1}^{\mathrm{y}} \mathrm{I}(\mathrm{x}, \mathrm{y})$

To save the image space, the integral average image is transformed within the image itself. If $g$ be the input image itself, self transformation is done with following equations as:

First raw : $g(1, y)=\frac{1}{y}[g(1 . c) \times c+g(1, y)]$

First column: $g(x, 1)=\frac{1}{x}[g(r, 1) \times r+g(x, 1)]$

Other points at $(x, y)$ :

$g(x, y)=\frac{1}{x \times y}[g(x, y)+g(r, y) \times r \times y+g(x, c) \times c \times x-$

$\mathrm{g}(\mathrm{r}, \mathrm{c}) \times \mathrm{r} \times \mathrm{c}]$

Where $\quad \mathrm{r}=\mathrm{x}-1, \mathrm{c}=\mathrm{y}-1, \mathrm{x}=2 . \mathrm{m}$ and $\mathrm{y}=2 . \mathrm{n}$. Using the above three equations Eqn (3-5), Eqn (2) can be implemented to transform a grey scale input image $g$ to an integral average image within itself. Its sequential diagram is shown in Figure 1. This figure shows the sequential steps (a-d) of transforming the input image $g$ into integral average image within itself using Eqns (3-5). Steps (a), (b) and (c) represent the corresponding tables. Lower values indicate the original values, whereas upper values with decimal points indicates the calculated average values. In the case of color images, average images are found for each channel R, G and B separately.

\subsection{Local sum and mean calculation}

Local $\operatorname{sum} \mathrm{s}(\mathrm{x}, \mathrm{y})$ at $(\mathrm{x}, \mathrm{y})$ within a block window of size $\mathrm{w} \times \mathrm{w}$ can be calculated from the integral average image $g$ with the following equation as

$\mathrm{s}(\mathrm{x}, \mathrm{y})=[\mathrm{g}(\mathrm{x}+\mathrm{d}, \mathrm{y}+\mathrm{d}) \times(\mathrm{x}+\mathrm{d}) \times(\mathrm{y}+\mathrm{d})+\mathrm{g}(\mathrm{x}-\mathrm{d}-$

$1, y-d-1) \times(x-d-1) \times(y-d-1)]-g(x-d-1, y+$

d) $\times(x-d-1) \times(y+d)+g(x+d, y-d-1) \times(x+d) \times$

$(\mathrm{y}-\mathrm{d}-1)]$

where $\mathrm{d}=\frac{\mathrm{w}-1}{2}$

From local sum $s(x, y)$, local mean $m(x, y)$ can be determined as

$m(x, y)=\frac{s(s, y)}{w \times w}$

\begin{tabular}{|c|c|c|c|c|c|c|c|c|}
\hline 2 & 4 & 5 & 3 & \begin{tabular}{|l|l|}
2.00 & \\
& 2
\end{tabular} & $3.00_{4}$ & 4.33 & 5 & 50 \\
\hline 3 & 8 & 3 & 6 & 3 & 8 & & 3 & 6 \\
\hline 5 & 2 & 1 & 4 & 5 & 2 & & 1 & 4 \\
\hline 4 & 4 & 5 & 6 & 4 & 4 & & 5 & 6 \\
\hline$a$ & & & & $b$ & & $\downarrow$ & & \\
\hline $2.00_{2}$ & $\begin{array}{|ll|}3.00 & \\
& 4\end{array}$ & \begin{tabular}{|l|}
4.33 \\
\end{tabular} & $\begin{array}{|ll|}4.50 & \\
& 3 \\
\end{array}$ & $2.00{ }_{2}$ & 3.00 & 4.33 & & 50 \\
\hline 2.503 & $\begin{array}{|ll|}4.25 & 8 \\
\end{array}$ & $\begin{array}{lll}4.50 & \\
& & 3 \\
\end{array}$ & \begin{tabular}{ll|}
4.75 & \\
& 6 \\
\end{tabular} & 2.50 & 8 & & 3 & 6 \\
\hline 3.335 & 4.00 2 & $\begin{array}{|ll|}3.89 & 1 \\
& 1\end{array}$ & $\begin{array}{|ll|}4.17 & \\
& 4 \\
\end{array}$ & 3.335 & 2 & & 1 & 4 \\
\hline 3.504 & $\begin{array}{lll}4.00 & \\
& 4\end{array}$ & 4.00 & 6 & 3.504 & 4 & & 5 & 6 \\
\hline
\end{tabular}

Figure 1: Integral average image transformation sequence

\section{LOCAL THRESHOLDING}

The binarized image $b(x, y)$ is found as

$$
b(x, y)= \begin{cases}0 & \text { if } I(x, y) \leq \pi(x, y) \\ 1 & \text { otherwise }\end{cases}
$$

where $\mathrm{I}(\mathrm{x}, \mathrm{y}) \in[0,1]$ be the intensity of a pixel at location (x, y) of the image I. In local adaptive technique, a threshold is calculated for each pixel, based on some local statistics of the neighborhood pixels within a block of size $w \times w$. Normally local techniques are time consuming to process the local statistics like local mean and standard deviation So as to minimize the computational time of local mean calculation, TR. Singh et al [15] propose an efficient way of determining local threshold by using Eqn.(9). They used integral sum image in Eqn.(1) as a prior process to determine the local sum to find local mean $\mathrm{m}(\mathrm{x}, \mathrm{y})$. But Integral sum image representation has a problem of large value of sum. To solve this problem, we propose integral average image representation as in Eqn (2). Local sum $\mathrm{s}(\mathrm{x}, \mathrm{y})$ and local mean $\mathrm{m}(\mathrm{x}, \mathrm{y})$ are determined using Eqns (6) and (7) respectively. This technique of thresholding use only mean while other techniques like Sauvola's [18] use both mean and standard deviation. The thresholding technique of T.R. Singh et al [15] to determine the local threshold value for local adaptation is given below.

$\mathrm{T}(\mathrm{x}, \mathrm{y})=\mathrm{m}(\mathrm{x}, \mathrm{y})\left\lceil 1+\mathrm{k}_{1}\left(\frac{\partial(\mathrm{x}, \mathrm{y})}{1-\partial(\mathrm{x}, \mathrm{y})}-1\right)\right\rceil$

where $\partial(\mathrm{x}, \mathrm{y})=\mathrm{I}(\mathrm{x}, \mathrm{y})-\mathrm{m}(\mathrm{x}, \mathrm{y})$ is the local mean deviation and $\mathrm{k}_{1} \in[0,1]$ is a bias which can control the level of adaptation varying threshold value. It controls the area of foreground and background in the binarised image resulting a convenient way of controlling contrast area.

The lower value of $\mathrm{k}_{1}$ makes the threshold value higher meaning the pixel becomes foreground and higher value of $\mathrm{k}_{1}$ lowers the threshold value, the pixel becomes background. With different values of $\mathrm{k}_{1}$, the adaptation level of threshold value can be adjusted. Based on this idea, the background 
region and foreground region take different role in the contrast stretching in enhancement technique.

\section{IMAGE TRANSFORMATIONS}

All Image enhancement simply means, transforming an image I into image $\mathrm{O}$ using $\mathrm{f}$, where $\mathrm{f}$ is the transformation function. The values of pixels in images $I$ and $O$ are denoted by $r \in[0,255]$ and $s \in[0,255]$, respectively. As said, the pixel values $r$ and $s$ are related by the expression

$s=f(r)$

where $f$ maps a pixel value $r$ into an another pixel value $s$. The results of this transformation are mapped into the grey scale range. So, the results are mapped into the range $[0, \mathrm{~L}-1]$, where $\mathrm{L}=2 \mathrm{k}, \mathrm{k}$ being the number of bits in the image being considered. So, for instance, for an 8-bit image the range of pixel values will be $[0,255]$.

\subsection{Power-Law Transformations}

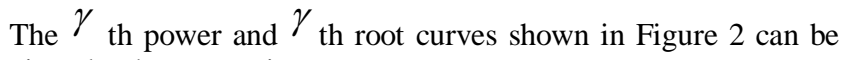
given by the expression:

$\mathrm{s}=\mathrm{cr}^{\gamma}$

This transformation function is also called as Gamma Correction (GC). For various values of $\gamma$ different levels of enhancements can be obtained. GC gives overall increase or decrease in intensity to output pixel value s. If the constant values $c$ and $\gamma$ are made dynamic, there will be a various level of enhancement unlike CG. T.R Singh et al [15] used this idea in adaptive power law transformation technique which is described in the next section.

\subsection{Adaptive Power-Law Transformations}

$\{I, O\} \in[0,1]$ are the input and output images. The Adaptive Power-law Transformations [14] is given by the following expression:

$s=c(1+k d) r^{\gamma(1-k d)}$

where $s \in[0,1]$ is the transformed enhanced output pixel value of image $O, r \in[0,1]$ is the input pixel of the image $I, c, \gamma$ and $k$ are constants which control the brightness, contrast and sharpness/smoothness of $O$.

$d=r-m(x, y)$

where $m(x, y)$ is the local mean of the input image within a window of size $w \times w$ with $r$ as centre at $(x, y)$ of the window.

If $k=0$, then it is equivalent to the GC and if $k \neq 0$, then the three parameters take different roles of varying different levels of enhancement of the output image unlike GC. There will be three controls depending on the different parameters such as brightness, contrast and sharpness/ smoothness of the output image. Calculation of local mean is time consuming and there will be unwanted contrast stretching for highly correlated region which is shown in Figure 3.(b). In this figure the black region indicates the more contrast, where white region indicates less contrast region. It includes the region where contrast is not necessary. This problem can be controlled by using a threshold vale like in Figure $3(\mathrm{c})$. Based on this condition a threshold based technique is proposed for replacing the mean $m(x, y)$ by a threshold value $T(x, y)$ in Eqn.(12).

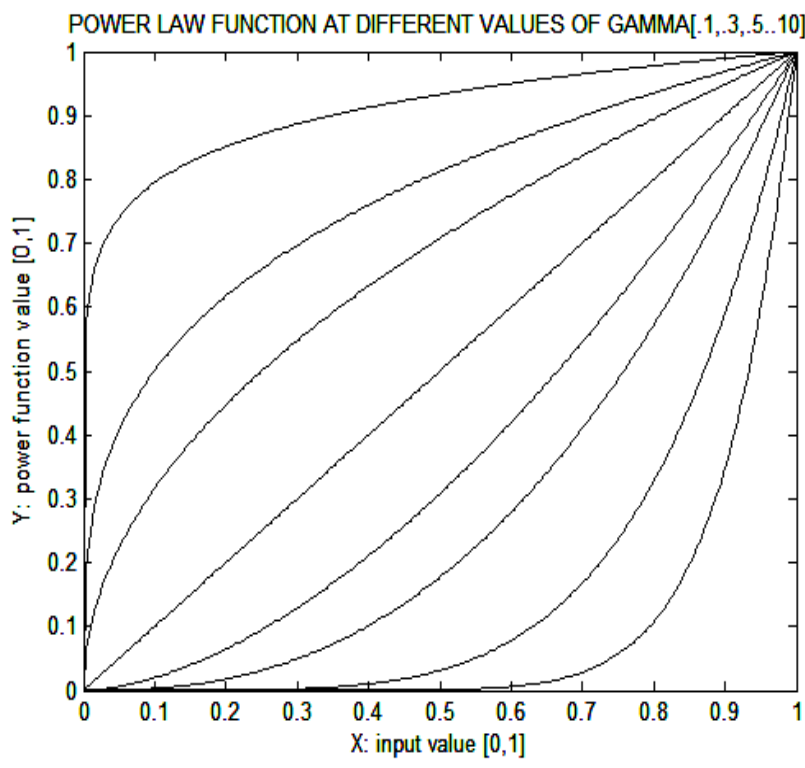

Figure 2 : Power Law Function at Different values of $\gamma \in\{0.1,0.3,0.5,1,1.7,2.5,5\}$

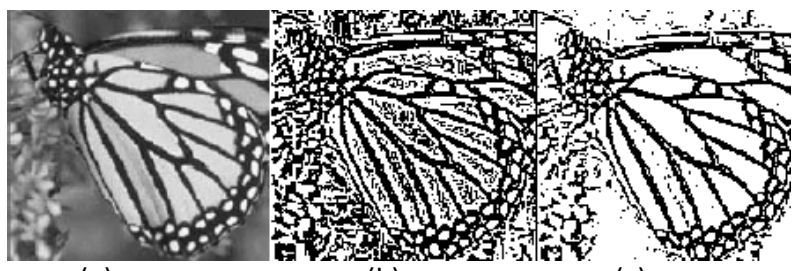

(a)

(b)

(c)

Figure 3: Contrast Region: (a) Original image. (b) contrast region by mean (c) contrast region by thresholding .

4.3 Adaptive Contrast Enhancement (ACE) Adaptive Contrast Enhancement (ACE) [12]-[19] is a wellknown local enhancement methods. It is expressed as:

$\mathrm{s}=\mathrm{m}(\mathrm{x}, \mathrm{y})+\mathrm{c}(\mathrm{r}-\mathrm{m}(\mathrm{x}, \mathrm{y}))$

where s, $\mathrm{r}$ are the output and input pixel value, $c$ is a contrast gain constant and $\mathrm{m}(\mathrm{x}, \mathrm{y})$ is local mean within a window of size $\mathrm{w} \times \mathrm{w}$. Using this constant $C$, all high-frequency components are amplified equally. As a consequence, those strong high frequency components will suffer from over enhancement.

\section{PROPOSED TECHNIQUE}

The Threshold based Adaptive Power-law Applications in image enhancement is similar to APLT[14] as in Eqn (12) and the difference is in Eqn (13). In Eqn (13) the mean $m(x, y)$ is replaced by a local threshold value $T(x, y)$ and it can be expressed as :

$\mathrm{d}=\mathrm{r}-\mathrm{T}(\mathrm{x}, \mathrm{y})$

where $T(x, y)$ is the local threshold value of pixels within a window of size $w \times w$ defined in Eqn.(9). $r$ is the concerned pixel at $(x, y)$ as centre of the window. Thus the 
proposed system is given by the Eqns (12) and (15). The new technique TAPLA is equivalent to ALPT if $\mathrm{k}_{1}=0$ in Eqn.(9). Like ALPT if $k=0$ it is same as GC and if $\mathrm{k} \neq 0$ there are various contrast adjustment levels depending on the value of $d$ with $k$ as a positive constant. Those pixel whose values are below the threshold $T(x, y)$, i.e. $d<0$ will give higher value of $\gamma(1-k d)$ and lower value of $c(1+k d)$ resulting lower transformed value of $s$. Those pixels whose values are greater than the threshold $T(x, y)$, i.e. $d>0$ will give lower value of $\gamma(1-k d)$ and higher value of $c(1+k d)$ resulting higher transformed value of $s$. If $k$ is negative constant, the result will be opposite resulting a smooth image output. Depending on the values of $\gamma$, there will be various levels of enhancement unlike APLT keeping the others parameters constant. As $d$ depends on $T(x, y)$ and $T(x, y)$ depends on $\mathrm{k}_{1}, \mathrm{k}_{1}$ also take a major role in this technique. Thus in this transformation technique, there may not be over all increase or decrease at the output values. The value of $d$ varies the level of contrast adjustment with the value of $\gamma$. The advantage of using Eqn. (15) is that it can control the contrast region according to the value of $k_{1}$ in Eqn. (9), whereas APLT is not there as in Figure 3. Figure 4 shows the various levels of contrast stretching of APLT and TAPLA. Not only the quality of image is enhanced, but also its computational time factor is reduced. Due to uses of integral average image, its computational time complexity is very low as compare with other adaptive techniques and it is closed to global techniques.

\section{EXPERIMENTAL RESULTS}

The proposed technique TAPLA is tested on many categories of images and compare with other spatial domain adaptive techniques like ACE and APLT qualitatively as well as quantitatively. The experiment was carried out using MATLAB 7.3 (R2006b) on a PC with the following configuration: Intel ${ }^{\circledR}$ Core ${ }^{\mathrm{TM}} 2 \mathrm{Duo}$ CPU E6550, GHz $2.33 \mathrm{GHz}, 2 \mathrm{~GB}$ RAM, 32 bit OS(Windows Vista).

\subsection{Image Evaluation}

Experimental results can be evaluated qualitatively or quantitatively according to the purpose. Qualitative evaluation provides a set of images for visual perception by human beings. It is highly subjective and it is not convenient to measure to quantify the enhancement for analysis. A quantitative measure is also needed in case of parameter based algorithms to identify the optimum enhancement point. When there is a need to compare the results of two enhancement techniques, a quantitative measure is highly imperative. Many measures of enhancement exist, but very few exhibit consistencies over all types of images. Many measures of enhancement do not show expected measurements in images exhibiting obvious visual contrast improvement. Our new technique associates contrast stretching, brightness as well as sharpness/smoothness and hence we choose the measure of enhancement by entropy (EME) [23-25] for evaluating our contrast enhancement results and Tenengrad measure is employed for evaluating edge information for sharpness.

EME is based on entropy of contrast established on the foundation of the Michelson contrast measure [19] and uses elements of human visual perception. It is expressed as

$E M E=\frac{1}{p \times q} \sum_{x=1}^{p} \sum_{y=1}^{q} \frac{I_{m}(x, y)}{I_{n}(x, y)+e} \log \left(1+\frac{I_{m}(x, y)}{I_{n}(x, y)+e}\right)$

where $\mathrm{p} \times \mathrm{q}$ is the total number of non-overlapping blocks of size $b \times b$ in the image $I_{m \times n}$ such that $p=\frac{m}{b}$ and $q=\frac{n}{b} I_{x}(x, y)$ and $I_{y}(x, y)$ are the maximum and minimum grey level value of pixels within the block $(\mathrm{x}, \mathrm{y}), \mathrm{e}$ is a very small constant added to the denominator to avoid division by zero. Higher value of EME denotes a higher contrast and information clarity in the image. Using a smaller block size $\mathrm{b} \times \mathrm{b}$ produces a more accurate result, but increases processing time. This measure of enhancement employs a more structured and rational approach than other measures to quantify contrast enhancement. It is a more reliable and accurate measure owing to the use of contrast entropy and other elements of visual perception. The measure shows considerable consistency and correctness that is more in conjunction with the visual quality and perceptibility of the image.

Tenengrad measure is based on gradient magnitude maximization. Tenengrad value of an image $I$ is calculated from the gradient $\operatorname{VI}(\mathrm{x}, \mathrm{y})$ at each pixel location $(\mathrm{x}, \mathrm{y})$, where the partial derivatives are obtained by a high pass filter like the Sobel operator, with the convolution kernels $i_{x}$ and $i_{y}$. The gradient magnitude is given as

$s(x, y)=\sqrt{\left(i_{x} \times I(x, y)\right)^{2}+\left(i_{x} \times I(x, y)\right)^{2}}$

Tenengrad criteria is then calculated as

TEN $=\sum_{\mathrm{x}} \sum_{\mathrm{y}} \mathrm{s}(\mathrm{x}, \mathrm{y})^{2}$

The image quality in terms of sharpness and edge information is usually considered higher if the TEN value is larger. In smoothening case TEN value is low for more smooth image as compare with the original image. Hence TEN value is depend on the evaluation purpose.

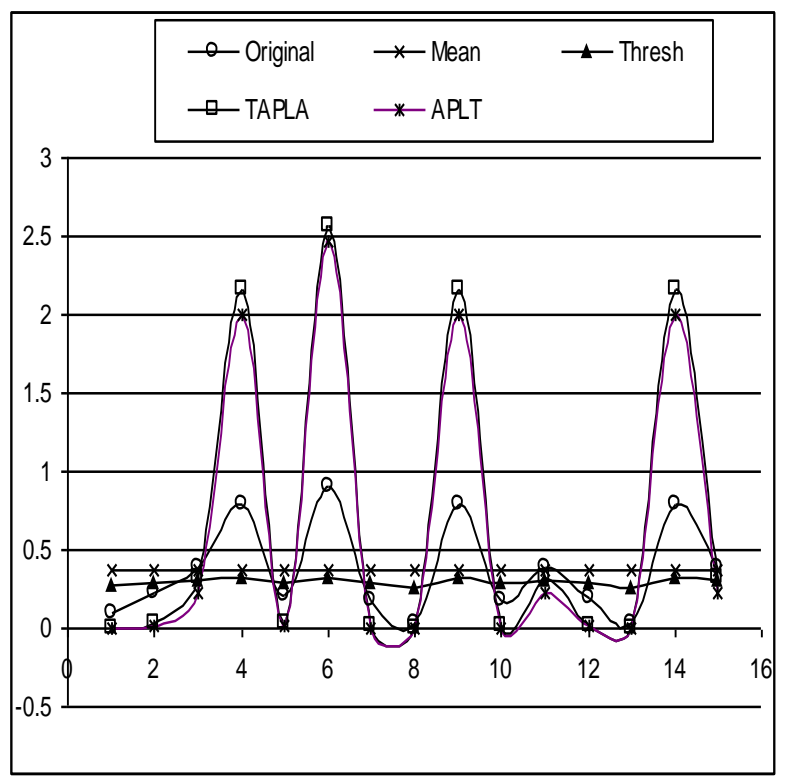

Figure 4: Different levels of contrast stretching by APLT and TAPLA with local mean and local threshold value. 


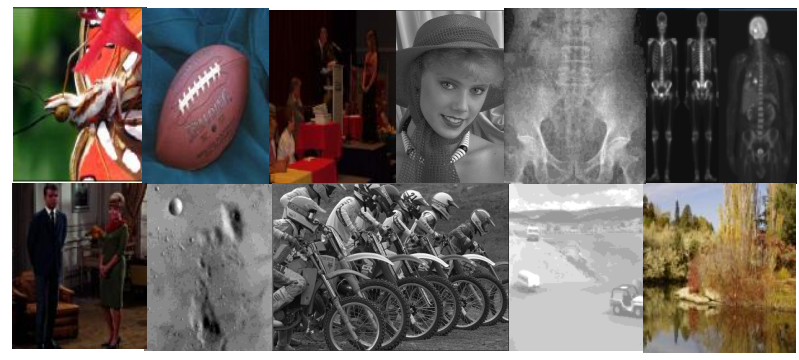

Figure 5: Images used in experiment.

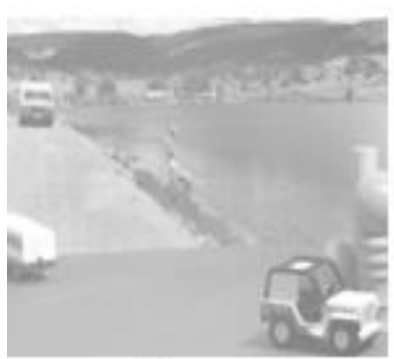

(a)

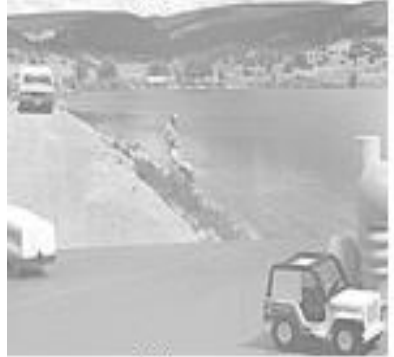

(c)

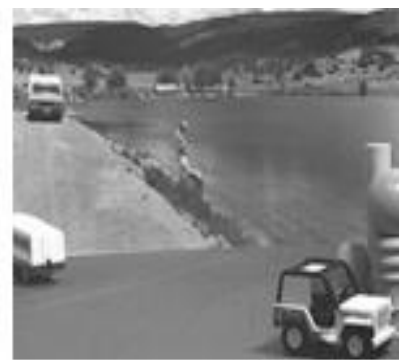

(b)

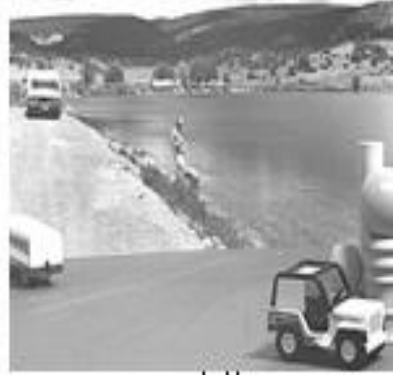

(d)
Figure 6: Visual comparison of output images of gray scene (469 $\times 346)$ : (a) Original, (b) APLT at $c=1, \gamma=2.5$ and $k=2$, (c)ACE at g=2, (d) TAPLA at $\mathrm{c}=1, \gamma=2.5, \mathrm{k}=2$, and $\mathrm{k}_{1}=0.06$.

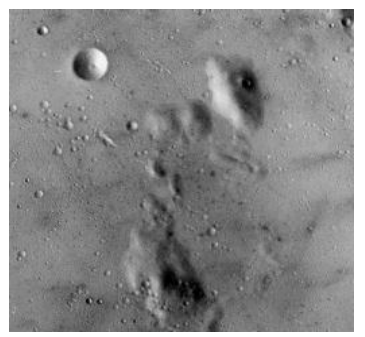

(a

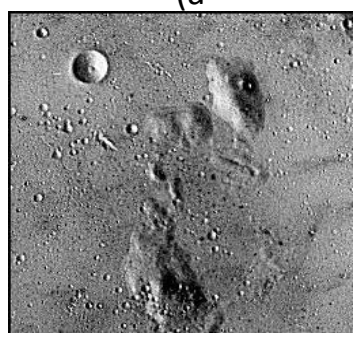

(c

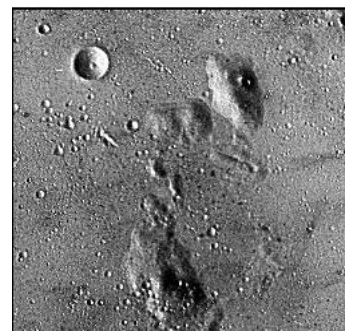

(b

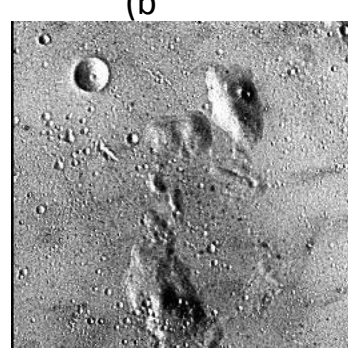

(d
Figure 7: Visual comparison of gray moon surface $(256 \times 256)$ : (a) Original, (b) APLT at $c=1, \gamma=1.1$ and $k=4$, (c)ACE at $\mathrm{g}=3$, (d) TAPLA at $\mathrm{c}=1, \gamma=1.1 \mathrm{k}=4$, and $\mathrm{k}_{1}=0.06$.

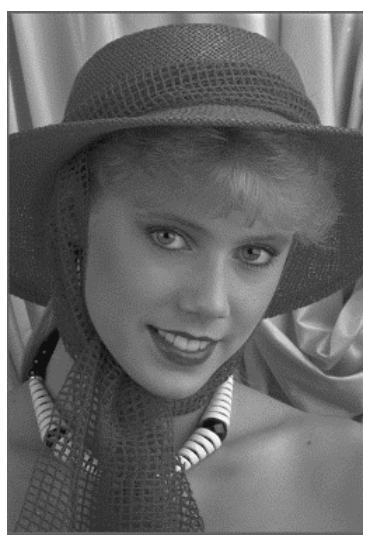

(a)

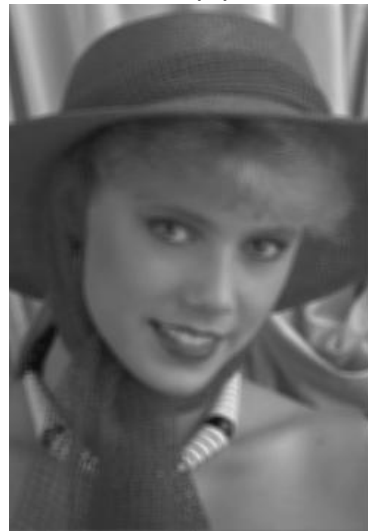

(c)

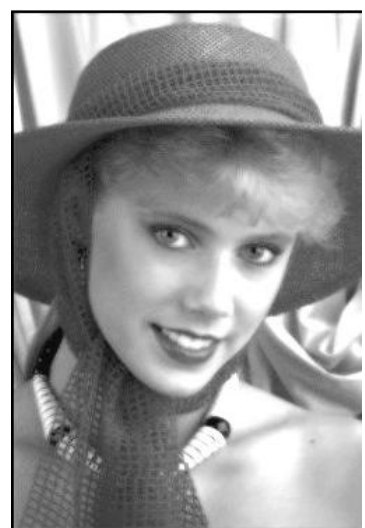

(b)

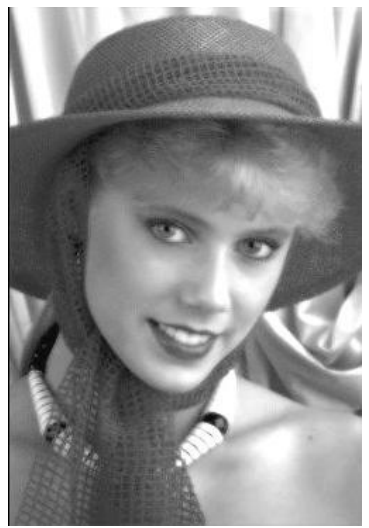

(d)
Figure 8: Visual comparison of output result images of Bele $(254 \times 384)$ : (a) Original, (b) APLT at $c=2, \gamma=1.5$ and $\mathrm{k}=-1$, (c)ACE at $\mathrm{g}=-0.1$, (d) TAPLA at $\mathrm{c}=2, \gamma=1.5$, $\mathrm{k}=-$ 1 , and $\mathrm{k}_{1}=0.1$

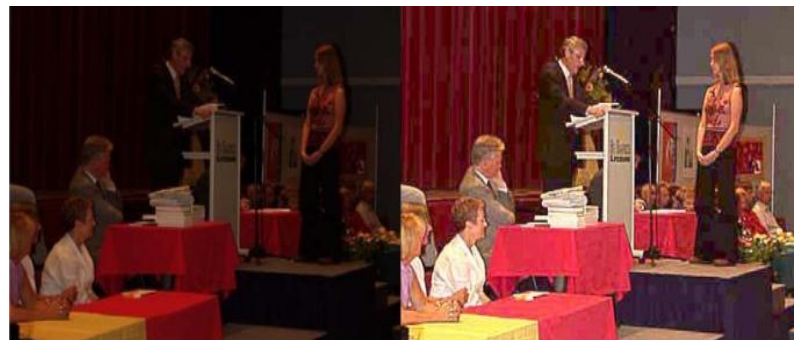

(a)

(b)

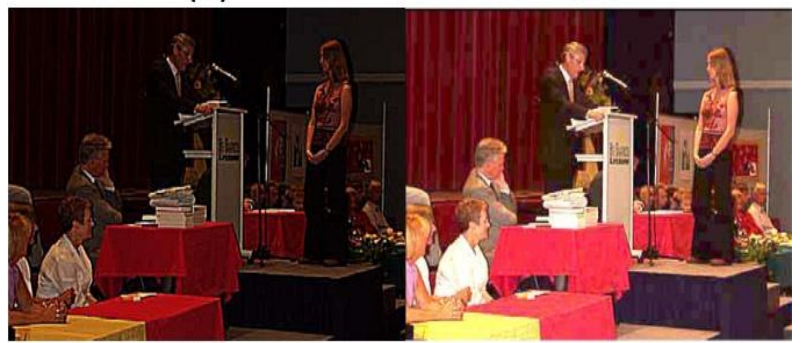

(c)

(d)

Figure 9: Visual comparison of output result of an dark image $(412 \times 310)$ : (a) Original, (b) Result by APLT at $\mathrm{c}=1.5, \gamma=0.56$ and $\mathrm{k}=2$, (c) ACE at $\mathrm{g}=2$, (d) Result by TAPLA at $c=1.5, \gamma=0569, k=2$, and $k_{1}=0.2$ 

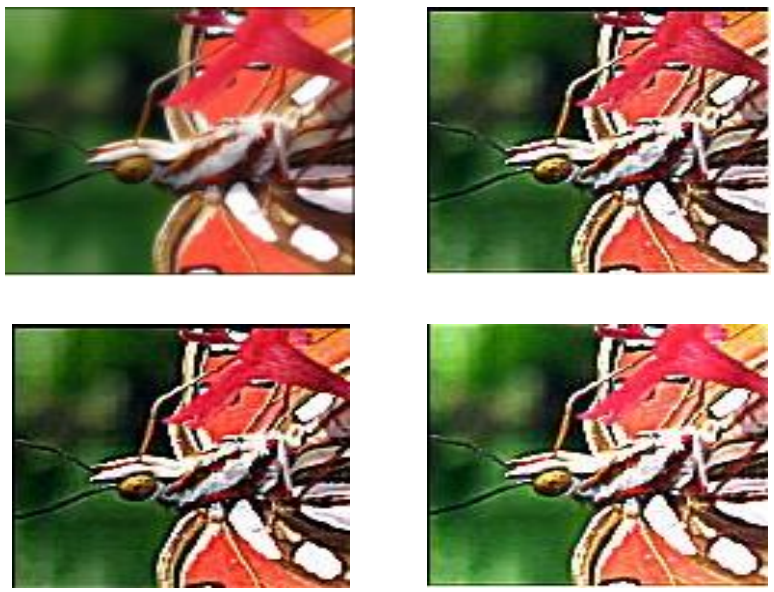

Figure 10: Visual comparison of output result images of Buterfly $(121 \times 122)$ : (a) Original, (b) APLT at $c=1, \gamma=0.9$ and $\mathrm{k}=2$, (c) ACE at $\mathrm{g}=2$, (d) TAPLA at $\mathrm{c}=1, \gamma=0.9$, $\mathrm{k}=2$, and $\mathrm{k}_{\mathbf{1}}=\mathbf{0 . 1}$

Table 1: EME Comparison of different methods

\begin{tabular}{|r|r|r|r|r|}
\hline \multirow{2}{*}{$\begin{array}{c}\text { Figure } \\
\text { No. }\end{array}$} & \multicolumn{4}{|c|}{ EME } \\
\cline { 2 - 5 } & \multicolumn{1}{|c|}{ Original } & \multicolumn{1}{|c|}{ APLT } & \multicolumn{1}{c|}{ ACE } & \multicolumn{1}{c|}{ TAPLA } \\
\hline 6 & 1.2 & 2000 & 2300 & 2400 \\
\hline 7 & 123 & 3900 & 3700 & 4300 \\
\hline 8 & 48.924 & 1080 & 4040 & 1110 \\
\hline 9 & 48.924 & 1080 & 4040 & 1130 \\
\hline 10 & 5.6072 & 3610 & 17400 & 3990 \\
\hline
\end{tabular}

Table 2: TAN Comparison of different methods

\begin{tabular}{|r|r|r|r|r|}
\hline \multirow{2}{*}{$\begin{array}{c}\text { Figure } \\
\text { No. }\end{array}$} & \multicolumn{4}{|c|}{ TAN } \\
\cline { 2 - 5 } & ORIGINAL & \multicolumn{1}{|c|}{ APLT } & \multicolumn{1}{l|}{ ACE } & TAPLA \\
\hline 6 & 2600 & 16000 & 13000 & 20000 \\
\hline 7 & 3470 & 41700 & 14100 & 61900 \\
\hline 8 & 454.1929 & 20800 & 1740 & 21400 \\
\hline 9 & 454 & 20800 & 1740 & 21700 \\
\hline 10 & 2620 & 7680 & 5800 & 8220 \\
\hline
\end{tabular}

Table3 : Computational Time Comparison different methods applied on Lena $512 \times 512$ image (in sec)

\begin{tabular}{|c|c|c|c|}
\hline $\begin{array}{c}\text { Window Size } \\
\boldsymbol{w}\end{array}$ & TAPLA & ACE & ALPT \\
\hline 3 & 0.2496 & 7.176 & 7.1448 \\
7 & 0.234 & 7.3944 & 7.3944 \\
11 & 0.234 & 7.9093 & 7.9561 \\
15 & 0.234 & 8.5177 & 8.5489 \\
19 & 0.234 & 9.2509 & 9.2821 \\
23 & 0.234 & 10.0777 & 10.0621 \\
27 & 0.2184 & 11.1073 & 11.0605 \\
31 & 0.2028 & 12.0745 & 12.1369 \\
35 & 0.1872 & 13.2913 & 13.3225 \\
\hline
\end{tabular}

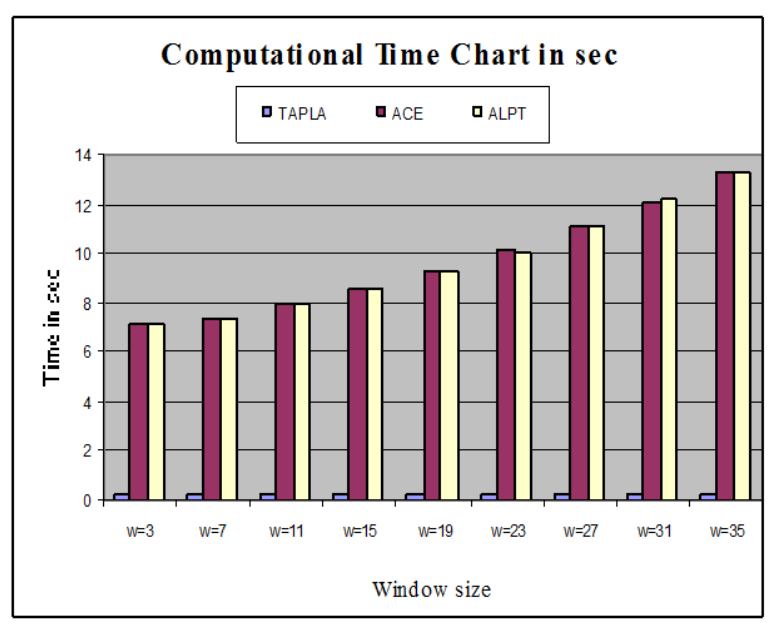

Fig. 11 Computational time chart of different methods at different window size applied on lena $512 \times 512$

\subsection{Result analysis}

The proposed technique TAPLA is tested on many categories of images like dark, bright and uniform luminance images of different types including medical image, scenery and aerial views. The test result is compared with other adaptive techniques like ACE and APLT. Figures 5 shows some of the tested images. Analysis is carried out through qualitative and quantitative measure of the output images. Figure (6-10) shows the results of different techniques for visual comparison. Figures (6-8) show the contrast stretching, edge sharpness and smoothness of gray scale images for different techniques respectively. Figures $(9,10)$ show the making of uniformed visualization of dark image and contrast as well as edge enhancement of color image respectively. Figures (1117) show the result of proposed technique TAPLA.

Quantitative analysis is also employed for this technique to compare with other techniques. Tables 1 and 2 show EME and TAN comparison with the other techniques. Higher the value of EME and TAN there is a high quality of contrast and edge information respectively. In the case of smoothing, EME and TAN are lower than the original one. In table 1 we found that ACE has higher value of EME which measure the contrast, in certain images like in Figures (8-10). But in the case of TEN which measure edge sharpness, TAPLA has higher value for all images which are shown in Figure (6-10) and it shown in table 2 .

For an algorithm, computational time complexity is a major factor. Normally adaptive techniques are local window size dependent and its computational complexity is $\boldsymbol{O}\left(\boldsymbol{w}^{2} \times \boldsymbol{n}^{2}\right)$ for an image of size $\boldsymbol{n} \times \boldsymbol{n}$ with local window size $\boldsymbol{w} \times \boldsymbol{w}$. But here in this proposed technique TAPLA, it is local window size independent as a result of using integral average image to determine local mean $m(x, y)$. Hence its computational time complexity is $\boldsymbol{O}\left(\boldsymbol{n}^{2}\right)$ which is very closed to global techniques. Figure 11 and Table 3 show the computational time comparison. Table 1 and 2 show the quantitative measures.

From the above experimental results we observe that this proposed technique TAPLA is outperform than the other local techniques. 


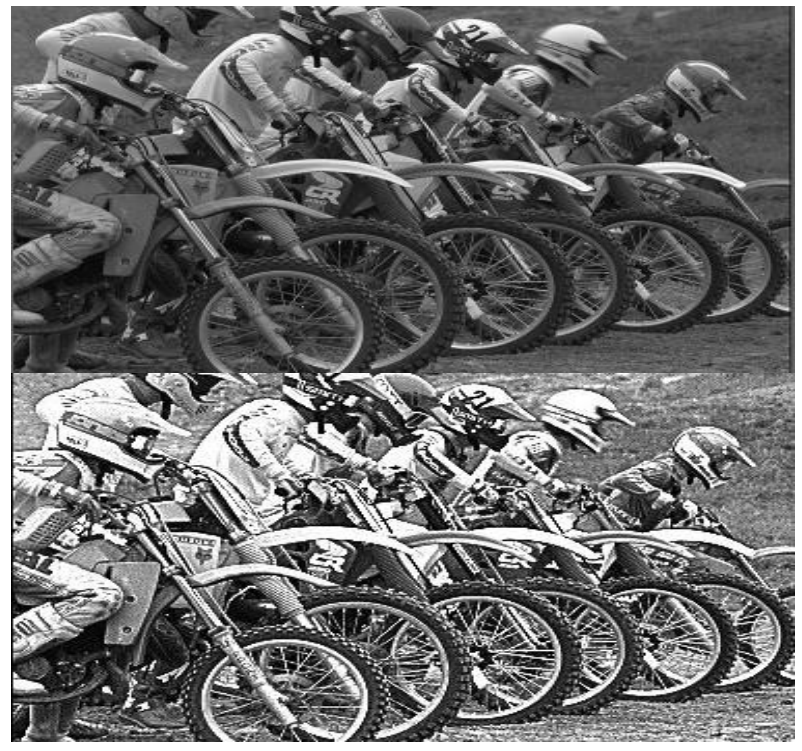

Figure 12: Output result images of a motor bike $(512 \times 512)$ by TAPLA at $c=2, \gamma=1.3$ and $k=2$, and $k_{1}=0.06$

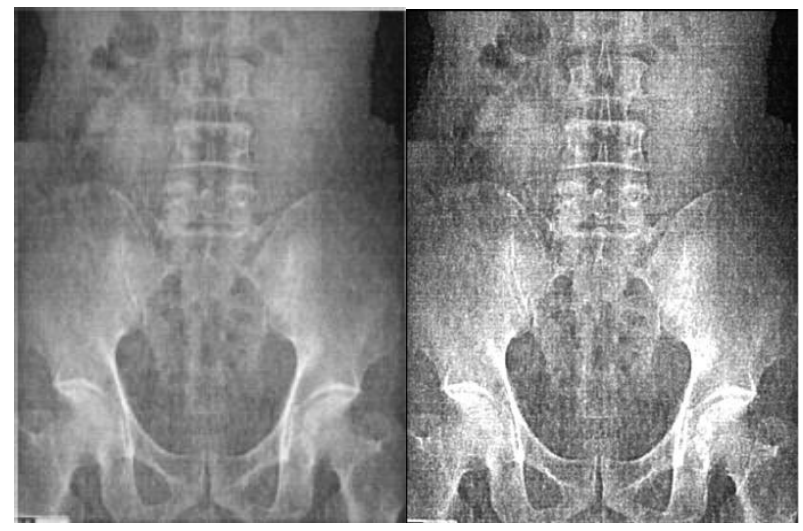

Figure 13: Output result of $x$-ray $(355 \times 479)$ by TAPLA at $c=1.2, \gamma=1.5$ and $k=2$, and $k_{1}=0.1$

\section{CONCLUSION}

This paper presents an improved version of adaptive power law transformations by replacing the local mean by local thresholding value which is determined using integral average image so as to determine local mean without depending on local window size. This new technique TAPLA outperform as compare with APLT and ACE both in image quality and computational time factor. But still this technique also has the problem of ringing edge artifact. TAPLA is a three parameter single function image enhancement technique. It can enhance an image through contrast, brightness and sharpness/smoothness with proper choice of respective parameters. Parameter choice is very important and if choosing of parameter adapts automatically based on the input image type, it should be much improve in image quality.

\section{ACKNOWLEDGMENTS}

We thank to all the authors regarding subject matter for giving us knowledge towards development of this paper in the field of image enhancement technique for publication to journal.

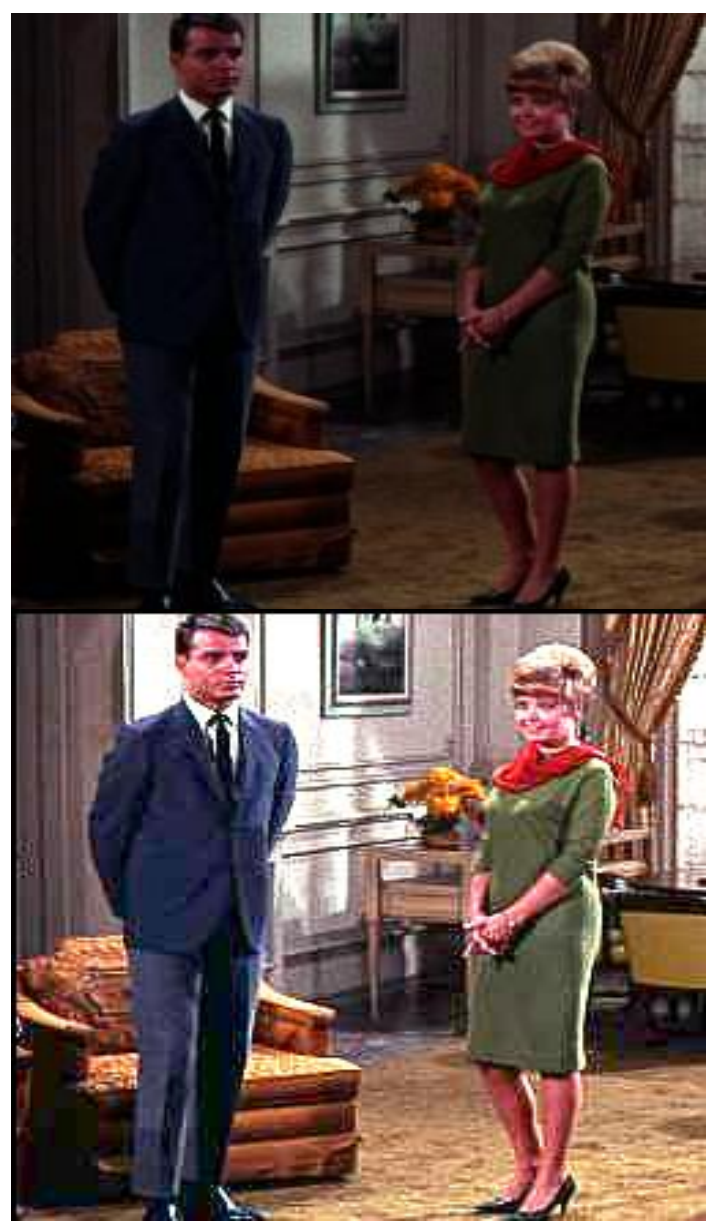

Figure 14: Output result images of room $(512 \times 512)$ by TAPLA at $\mathrm{c}=2, \gamma=.8$ and $\mathrm{k}=2$, and $\mathrm{k}_{1}=0.06$

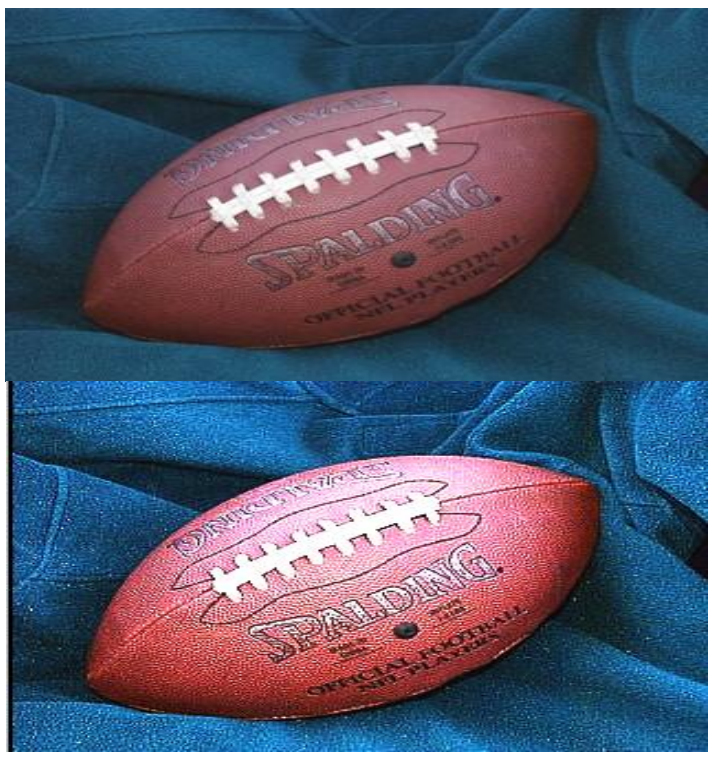

Figure 15: Output result images of a ball $(512 \times 512)$ by TAPLA at $\mathrm{c}=2, \gamma=1.3$ and $\mathrm{k}=2$, and $\mathrm{k}_{1}=0.06$. 


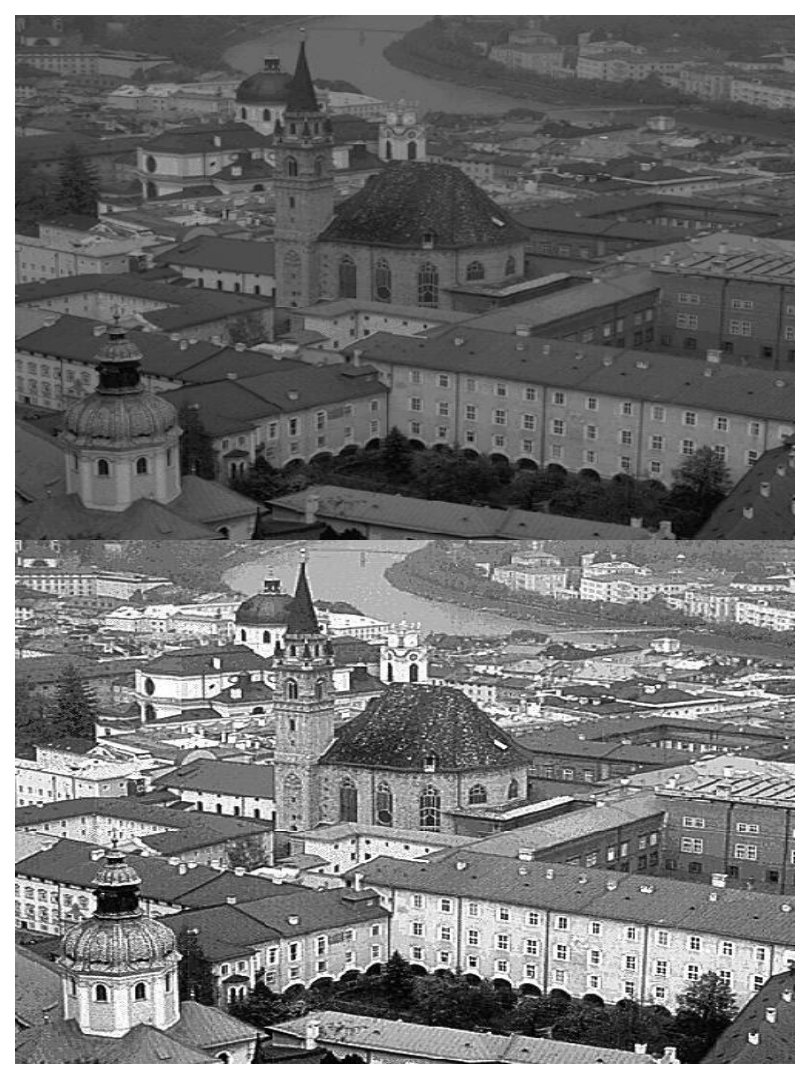

Figure 16: Output result images of a Tample $(512 \times 512)$ by TAPLA at $c=2, \gamma=1.3$ and $k=2$, and $k_{1}=0.06$

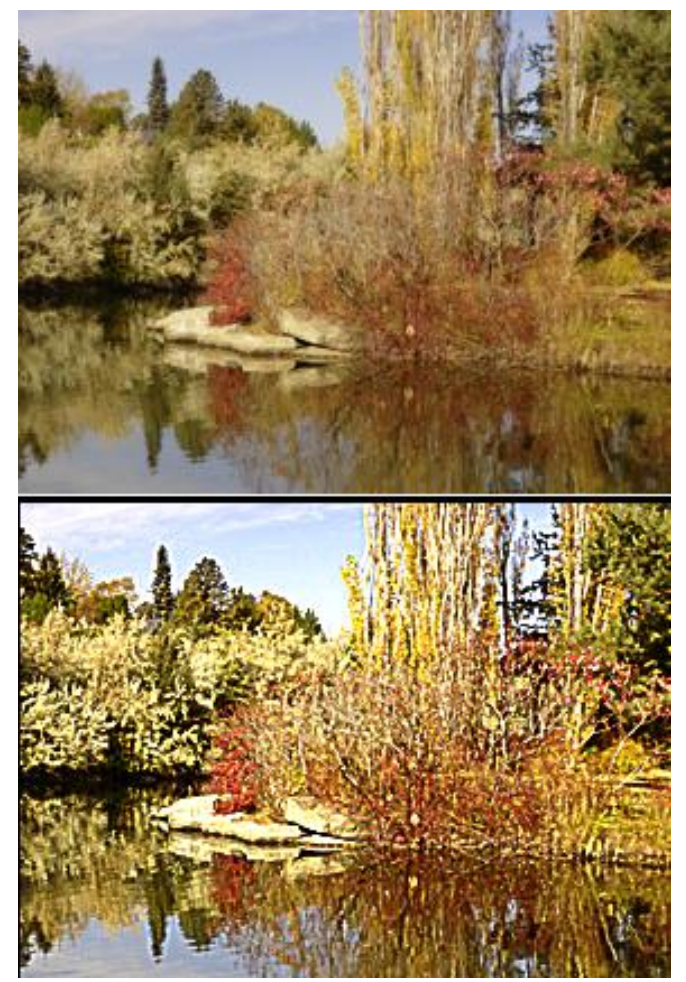

Figure 17: Output result images of a scenery $(480 \times 480)$ by TAPLA at $c=1.2, \gamma=1.2$ and $k=3$, and $k_{1}=0.1$

\section{REFERENCES}

[1] R.C. Gonzales, R. E. Woods, Digital Image Processing, $2^{\text {nd }}$ Edn. 2005.

[2] S. Mallat, Characterization of signals for multiscale edges, IEEE Trans. Patt. Anal., Machine Intelligence, vol. PAMI 14, pp. 710-732, 1992.

[3] J.K. Kim, J.M. Park, K.S. Song and H.W. Park, Adaptive mammographic image enhancement using first derivative and local statistics, IEEE Trans. Medical Imaging, vol. 16, iss. 5, pp. 495-502, Oct. 1997.

[4] J.L. Starck, E. Cand es, and D.L. Donoho. The curvelet transform for image denoising. IEEE Transactions on Image Processing, 11(6):131\{141, 2002.

[5] Nagesha and G.H. Kumar, A level crossing enhancement scheme for chest radiograph images, Elsevier, Computer in Biology and Medicines, vol. 37, iss. 10, pp. 14551460, Oct 2007.

[6] Kuroda, Algorithm and architecture for real time adaptive image enhancement, SiPS 2000, pp. 171-180, 2000.

[7] D.C. Chang and W.R. Wu, Image contrast enhancement based on a histogram transformation of local standard deviation, IEEE Trans. MI, vol. 17, no. 4, pp. 518-531, Aug. 1998.

[8] G. Boccignone and M. Ferraro, Multiscale contrast enhancement, Electron. Lett., vol. 37, no. 12, pp. 751752, 2001.

[9] Sarif Kumar Naik and C. A. Murthy "Hue-Preserving Color Image Enhancement Without Gamut Problem", IEEE Transactions On Image Processing, Vol. 12, No. 12, December 2003.

[10] R.N. Strickland, C.S. Kim and W.F. Mcdonnel, Digital color image enhancement based on the saturation component, Opt. Engg, vol. 26, no. 7, pp. 609-616, 1987.

[11] J. S. Lee, "Digital image enhancement and noise filtering by using local statistics," IEEE Trans. Pattern Anal. Machine Intell., vol. PAMI-2, pp.165-168, Feb. 1980..

[12] T.-L. Ji, M. K. Sundareshan, and H. Roehrig, "Adaptive image contrast enhancement based on human visual properties," IEEE Trans. Med. Imag., vol. 13, pp. 573586, Aug. 1994.

[13] L. Lucchese, S. K. Mitra, and J. Mukherjee, "A new algorithm based on saturation and desaturation in the $\mathrm{xy}$ chromaticity diagram for enhancement and re-rendition of color images," Proc. IEEE Int. Conf. on ImageProcessing, pp. 1077-1080, 2001.

[14] T.Romen Singh, O.Imocha Singh , Kh. Manglem Singh , Tejmani Sinam and Th. Rupachandra Singh "Image Enhancement by Adaptive Power-Law Transformations", Bahria University Journal of Information and Communication Technology Volume 3 Issue 1 (BUJICT 2010), ISSN 1999-4974.

[15] T.Romen Singh, Sudipta Roy , O.Imocha Singh, Tejmani Sinam, Kh. Manglem Singh "A New Local Adaptive Thresholding Technique in Binarization", IJCSI International Journal of Computer Science Issues, Vol. 8, Issue 6, No 2, November 2011 ISSN (Online): 16940814 . 
[16] Bernsen, J. 1986, Dynamic thresholding of gray-level images. Proc. $8^{\text {th }}$ Int. Conf. on Pattern Recognition, Paris, pp $1251-1255$

[17] W. Niblack, 1986 An Introduction to Image Processing,Prince Hall Englewood Cliffs, NJ.

[18] J. Sauvola and M. Pietikainen, "Adaptive document image binarization,” Pattern Recognition 33(2), pp 255-236,2000.

[19] Jinshan Tang, A contrast based image fusion technique in the DCT domain, Digital Signal Processing 14(2004) 218226.

[20] Iyad Jafar and Hao Ying, A New Method for Image Contrast Enhancement Based on Automatic Specification of Local Histograms, IJCSNS International Journal of Computer Science and Network Security, VOL. 7, July 2007.

[21] Bei Tang, Guillermo Sapiro ,Color Image Enhancement via Chromaticity Diffusion, IEEE TRANSACTIONS ON IMAGE PROCESSING, VOL. 10, NO. 5,May,2001.

[22] Agaian, S. S, K. Panetta A. M. Grigoryan : A new measure of image enhancement. In: IASTED Int. Conf. Signal Processing Communication, Marbella, Spain, Sep,1922,2000 .

[23] Agaian, S. S., K. Panetta, A. Grigoryan. : Transform based image enhancement with performance measure. In: IEEE Transactions on Image Processing, vol. 10,No. 3, pp.367381, March,2001.

[24] Agaian, Sos S., Blair Silver, Karen A. Panetta. : Transform Coefficient Histogram-Based Image Enhancement Algorithms Using Contrast Entropy. In: IEEE Transactions on Image Processing, vol. 16, No. 3,March, 2007.
[25] Silver, B., S. S. Agaian, K. A. Panetta. : Logarithmic transform coefficient Histogram matching with spatial equalization. In: SPIE Defence and Security Symposium, Mar. 2005.

[26] Narasimhan K, Sudarshan C R and Nagarajan Raju, A Comparison of Contrast Enhancement Techniques in Poor Illuminated Gray Level and Color Images, International Journal of Computer Applications (0975 8887) Volume 25- No.2, July 2011.

[27] Sos Agaian, Blair Silver》, and Karen Panetta,, Transform Coefficient Histogram Based Image Enhancement Algorithms using Contrast Entropy, TIP-01692-2005.

[28] Konstantinos G. Derpanis," Integral image-based representations", Viola, P. \& Jones, M. (2001). Rapid object detection using a boosted cascade of simple features. In IEEE Computer Vision and Pattern Recognition (pp. I:511-518).

[29] Dah-Chung Chang* and Wen-Rong Wu, Image Contrast Enhancement Based on a Histogram Transformation of Local Standard Deviation, IEEE TRANSACTIONS ON MEDICAL IMAGING, VOL. 17, NO. 4, AUGUST 1998.

[30] Sascha D. Cvetkovic*^, Johan Schirris*, Peter H.N. de With , Locally-Adaptive Image Contrast Enhancement Without Noise And Ringing Artifacts , 1-4244-14377/07/\$20.00 @2007 IEEE. 